Recherches en didactique des langues et des cultures

Les cahiers de l'Acedle

18-1 | 2021

En quoi les langues ont-elles un rôle à jouer dans les sociétés mondialisées au sein d'une Europe fragilisée ?

\title{
L'exposé oral en langue étrangère : une tâche complexe au service du développement des compétences
}

Marcelo Tano

\section{OpenEdition}

Journals

Édition électronique

URL : https://journals.openedition.org/rdlc/8714

DOI : $10.4000 /$ rdlc. 8714

ISSN : $1958-5772$

Éditeur

ACEDLE

\section{Référence électronique}

Marcelo Tano, «L'exposé oral en langue étrangère : une tâche complexe au service du développement des compétences », Recherches en didactique des langues et des cultures [En ligne], 18-1 | 2021, mis en ligne le 30 juin 2021, consulté le 03 août 2021. URL : http://journals.openedition.org/rdlc/8714 ; DOI : https://doi.org/10.4000/rdlc.8714

Ce document a été généré automatiquement le 3 août 2021.

\section{cc) (†) $\odot$}

Recherches en didactique des langues et des cultures is licensed under a Creative Commons AttributionNonCommercial-NoDerivatives 4.0 International License 


\title{
L'exposé oral en langue étrangère : une tâche complexe au service du développement des compétences
}

\author{
Marcelo Tano
}

\section{Introduction}

1 Selon les conclusions d'un récent sondage d'envergure réalisée auprès d'un échantillon de de 2084 étudiants de l'enseignement supérieur, la maîtrise de l'expression orale en langue étrangère (dorénavant LE) est un «[...] atout majeur qui assure le bon déroulement de la formation universitaire, l'intégration sociale et la future employabilité des étudiants» (Tano, 2021: 20). Or, l'une des grandes difficultés rencontrées à l'université est de concrétiser l'objectif communicationnel assigné à l'apprentissage des LE, qu'elles soient générales ou de spécialité. Ceci est tout particulièrement vrai pour l'expression orale en continu qui prend la forme d'un exposé oral (dorénavant EO) en présentiel offrant l'occasion de réaliser un entraînement à la prise de parole en public.

2 Dans le cadre de la présente étude, notre problématique centrale est la suivante : étant donné les difficultés habituellement observées sur le terrain par les étudiants universitaires lors de la préparation et de la présentation d'un EO en LE, ne serait-il pas profitable, tant dans la perspective de l'enseignement que dans celle de l'apprentissage, d'aborder cette production orale de façon plus explicite?

Nous partons du principe que prendre la parole en public est une pratique exigeant des techniques qui s'apprennent, surtout lorsque cette pratique se fait en LE. L'adoption d'une méthodologie plus guidée et plus séquencée, basée sur les théories de l'enseignement explicite, aiderait davantage les étudiants à acquérir les savoir-faire procéduraux nécessaires pour la préparation d'un EO. Cette procéduralisation est une phase associative de l'apprentissage dont, en suivant Musial et al (2021:7), nous dirons qu'elle consiste «à associer un but, une situation et une procédure ». Ces auteurs 
(Ibidem) précisent : "C'est le processus de transformation de quelque chose que l'on parvient à réaliser par tâtonnement en quelque chose que l'on sait faire de façon explicite et contrôlée ». C'est précisément parce que l'étudiant connaît de façon claire ce qu'il lui revient de faire pendant les différentes étapes de l'EO que la tâche devient enrichissante et source d'apprentissage.

Nous situons l'EO dans une pratique pédagogique pertinente à la lumière des théories socioconstructiviste et cognitiviste. Selon ces approches (Gautier, 2017 : 231), l'étudiant apprend en restructurant constamment ses connaissances et en intégrant de nouvelles informations à celles qu'il a déjà assimilées pour construire son propre savoir de façon à la fois personnelle et collective. En effet, l'EO étant d'abord une production individuelle sur un sujet à priori librement choisi, il ne prend son sens que parce que la présentation est faite en public et qu'elle est partagée avec la classe. C'est pour cette raison qu'il représente souvent un «[...] espace de liberté valorisant pour les élèves » (Zahnd, $1998:$ 43).

5 Puisque des difficultés peuvent apparaître lors de cette présentation, il est fondamental que l'apprentissage devienne quelque chose de totalement conscient pour l'étudiant. Cette pédagogie explicite s'inspire d'une stratégie " [...] favorisant une conscientisation des objectifs et des moyens, structurée en étapes séquencées - le découpage d'une tâche complexe en sous-tâches évitant une surcharge cognitive » (Gautier, $2017: 233$ ). L'activité de production orale ainsi conçue vise donc la prise de conscience des étapes nécessaires à une bonne préparation et présentation de l'EO.

La méthodologie s'inspirant de la pédagogie explicite (Id. : 240) et visant l'acquisition d'automatismes semble parfaitement adaptée à l'EO. Cette méthodologie est répétitive parce qu'elle se fait de façon similaire pour tous les EO. Si l'activité se répète tout au long d'un semestre, par exemple, les étudiants pourront profiter des techniques de leurs pairs en vérifiant ce qui a marché (ou pas!) pour ces derniers. La répétition de cette tâche complexe sert donc à installer des savoirs et des savoir-faire capitalisables et donc réutilisables. Ainsi, une première prestation constitue une phase de modelage et la répétition du modèle méthodologique faite par d'autres étudiants dans les EO qui suivront forme, selon Gautier (2017: 241), la "pratique guidée ». Parallèlement, le caractère évolutif est évident dans la mesure où l'approche proposée devient de moins en moins guidée étant donné que la méthodologie est de plus en plus connue par les étudiants. Cette procéduralisation progressive crée donc de l'autonomisation que cette auteure appelle « la pratique autonome ». Toutes ces idées nous amènent à considérer que la réussite d'un EO est fortement subordonnée à la conscience méthodologique.

7 Nous pensons qu'il devient urgent de dynamiser l'oral en milieu universitaire où l'accent est souvent mis sur les activités de compréhension et d'expression écrites. Force est de constater que les difficultés commencent quand on essaie de pondérer le degré attendu de spontanéité, de fluidité et de correction linguistique exigé dans un EO. Rappelons que l'expression spontanée est le résultat d'une longue préparation et que muscler sa maîtrise de l'oral n'est pas possible sans entraînement car prendre la parole et affronter le public est un savoir-faire qui s'apprend. Par conséquent, il est impossible de se passer d'un véritable travail didactique et régulier de l'EO car le bagage technique des étudiants dans ce domaine peut faire défaut à cause de la méconnaissance notamment des aspects méthodologiques qui préfigurent ce genre de compétence discursive. 
8 Les réflexions que nous proposons s'appuient sur deux hypothèses : i) l'EO peut devenir une activité stimulante de nature à donner l'envie de surmonter les obstacles et diminuer le niveau de lathophobie ${ }^{1}$, et ii) l'enseignement-apprentissage de l'oral doit être étayé par des pratiques explicitées et maîtrisées permettant de s'attaquer aux difficultés identifiées et facilitant le développement de certaines compétences langagières propres à l'oralité.

Afin de vérifier ces hypothèses, nous allons d'abord prendre appui sur les fondements théoriques qui sous-tendent l'EO en LE en esquissant ses traits définitoires. Nous allons ensuite aboutir à un modèle d'application viable et explicite qui tienne compte des étapes incontournables pour mener à bien cette tâche complexe. En questionnant un groupe d'étudiants bien identifiés, nous obtiendrons leurs points de vue concernant la façon dont ils font face à cette tâche et les bénéfices qu'ils pensent en tirer. Après l'analyse des principales tendances qui se dégagent de l'enquête ad hoc administrée, nous allons proposer quelques conclusions qui ouvriront certainement des perspectives pour des recherches futures.

\section{Les fondements théoriques de l'exposé oral en langue étrangère}

\subsection{Les traits définitoires de l'exposé oral en tant qu'objet d'enseignement-apprentissage}

10 Sur un plan pédagogique, l'EO est une courte présentation parlée, ordonnée et méthodique, d'un sujet imposé ou non, dont le but est de faire utiliser au maximum la LE en exposant aux autres membres du groupe-classe un thème sur lequel ils n'ont peut-être pas de connaissances. Remarquons que l'EO, se situant chronologiquement avant le débat ou la discussion, s'appuie sur la notion d'" expression orale en continu » étudiée dans le Cadre Européen Commun de Référence pour les Langues (Conseil de l'Europe, 2001 : 49-50) qui propose des grilles d'évaluation allant du niveau A1 à C2. Plus récemment, dans sa partie «s'adresser à un auditoire », le Volume Complémentaire du CECRL (Conseil de l'Europe, $2018:$ 77) a complété les descripteurs initialement fournis pour déterminer le niveau de compétence. Il découle de ce référentiel que plus le niveau augmente, plus les capacités langagières se déploient dans l'accomplissement d'une tâche de production si exigeante comme celle d'un EO.

11 Cette prise de parole autonome se fait dans une situation de communication qui permet à l'étudiant d'installer des connaissances dans le groupe-classe tout en incitant à la pratique de la LE. Bronckart et Dolz (1999: 39) définissent l'EO comme «[...] une prise de parole en public qui développe de manière explicitement structurée un sujet de l'ordre du savoir ». Cette notion d'explicitation construite est aussi développée par Dolz et Scheuwly (1998: 142) pour qui l'EO serait « un genre textuel public, relativement formel et spécifique, dans lequel un exposant expert s'adresse à un auditoire, d'une manière (explicitement) structurée, pour lui transmettre des informations, lui décrire ou lui expliquer quelque chose ". Les deux auteurs qualifient l'EO comme étant une activité qui se définit par son caractère largement monologal où l'énonciateur prend le rôle d'expert sur le thème développé. 
12 Les travaux de Vaissière (1999: 6-16) sur la prosodie ou phonétique de la phrase, montrent bien que l'oral se distingue par son caractère prosodique, c'est-à-dire, relatif à l'ensemble des phénomènes variés et inséparables du discours comme la qualité de la voix, la mélodie, la courbe intonative, le ton, le volume ou l'intensité, les accents d'insistance, le rythme, la durée, les pauses, etc.

13 L'EO s'appuie aussi sur les aspects propres à la communication non-verbale qui occupe une place non négligeable car la capacité de joindre le geste à la parole est mise à rude épreuve dans ce genre d'activité. Les gestes accompagnent les activités langagières et produisent de nombreux effets, à tel point qu'Alrabadi (2011:21-22) préconise la prise en compte de la proxémie pour la gestion des activités orales. Ceci s'explique parce que, en plus des traits prosodiques, le comportement paralinguistique comprend aussi le langage du corps (Conseil de l'Europe, 2001: 73) à travers les mouvements, les expressions du visage, la posture, le contact oculaire et la proximité. À cela s'ajoutent certains aspects visuels de l'EO qui relèvent des éléments paratextuels des textes écrits, tels que (Ibidem): l'illustration (photographies, dessins, les tableaux, schémas, diagrammes et figures, etc.) et la typographie (gras, italiques, espacement, soulignement, marges, etc.).

Dans cette optique, l'analyse de l'EO doit être faite non seulement en tenant compte des connaissances linguistiques mais aussi du rôle de la gestualité. Kerbrat-Orecchioni (1996: 45) rappelle que " la mimogestualité a pour l'émetteur une fonction de facilitation cognitive, c'est-à-dire qu'elle l'aide à effectuer les opérations d'encodage ». L'interaction voix/corps est par conséquent une spécificité de l'activité que nous décrivons. Pour Manolescu (2013: 111), l'expression orale englobe

«[...] le non-verbal (sous forme de gestes, de signes, de sourires, d'expressions gestuelles diverses adaptées à la situation de communication), la voix (volume, articulation des sons, débit de la voix ou de l'intonation pour que la communication soit faite de manière expressive), les pauses, les silences, les regards (pour vérifier le niveau de compréhension du message verbal) ».

Miecznikowski et al (2001: §1), plaçant l'EO comme une modalité de communication fréquente en milieu professionnel, le circonscrivent de la manière suivante :

« L'exposé est une activité pratique et interactive située au cours de laquelle il s'agit de gérer un temps limité de parole, de tenir compte des particularités de l'audience, d'être adéquat au contexte, de s'appuyer sur des aide-mémoires tels que les notes préparatoires et de présenter des documents écrits et visuels au public comme aides à la réception, administration de preuves, exhibition d'évidences ».

16 À partir de ces explications, nous pouvons déceler certaines caractéristiques définitoires de l'EO. Il s'agit d'un événement : i) contingent (il se déroule sous forme séquentielle dans un temps et dans un lieu précis, dans des circonstances contextuelles qui le déterminent); ii) préparé (il se base sur des notes et des textes préparatoires); iii) outillé (il comporte le maniement de ressources matérielles intermédiaires à caractère scriptovisuelles); iv) transmissif et à visé didactique (l'orateur fait progressivement émerger un savoir à travers son activité cognitive); et v) interactionnel (l'exposant s'oriente vers son public et interagit avec lui).

$17 \mathrm{Au}$ vu de ce qui précède, nous partons du principe que, si l'expression orale s'enseigne, l'EO peut donc représenter une situation envisageable dans les pratiques de classe et une activité utile pour développer la maîtrise des compétences langagières. De notre point de vue, un véritable travail didactique s'impose si nous voulons minimiser l'impact des écueils que l'étudiant doit surmonter pour réaliser cette activité. Afin de 
mettre en œuvre des stratégies concrètes et des procédures explicites d'intervention, nous estimons que l'enseignement de l'expression orale par l'intermédiaire de l'EO nécessite l'interface d'un modèle didactique qui détermine les caractéristiques et les dimensions enseignables et apprenables de ce genre discursif.

\subsection{Vers un modèle didactique explicite de l'exposé oral en langue étrangère}

Pour être en conformité avec les approches communicatives en vigueur en didactique des langues-cultures (DLC), tout enseignement-apprentissage de l'oral doit être étayé par l'insertion de différents genres oraux parmi lesquels les plus significatifs sont la conversation, la discussion, le débat, l'interview, la plaidoirie, la lecture à haute voix et, naturellement, l'EO. Chacun de ces genres a ses propres objectifs et obéit à des règles et techniques différentes et parfois complémentaires. Maintenant, s'ils sont au cœur des préoccupations en matière d'acquisition d'une LE à l'oral, Crosnier et Décuré (2018 : \$3) précisent que l'initiative de la prise de parole devant le groupe paraît avoir été peu explorée dans le domaine de la DLC.

Pour travailler des compétences visant à l'amélioration formelle de la parole, il faudrait installer un travail sur l'oral comme activité centrale où la pratique de l'EO munisse l'étudiant d'un répertoire verbal structuré, riche et diversifié et d'une maitrise progressive des supports technologiques. L'EO ne peut constituer un entraînement efficace que si les étudiants sont conscients des "savoir-faire procéduraux " (Richer, 2014: 38) requis pour effectuer des tâches qui exigent une grande rigueur méthodologique.

Il nous semble que le modèle didactique de l'EO prend du sens par l'intégration de deux concepts majeurs de la pédagogie explicite, celui de tâche complexe et celui d'apprentissage par étapes. L'EO est, en effet, une tâche complexe qui, selon Bourguignon (2006: 63) sous-tend la mobilisation de nombreuses ressources cognitives et méthodologiques. D'après Nissen $(2011: \$ 42)$ « une tâche complexe est celle qui comporte des sous-tâches, que l'on peut encore appeler étapes». La tâche est dite " complexe » non en raison de sa difficulté ${ }^{2}$ mais à cause des nombreuses étapes qu'il faut suivre pour l'accomplir. L'objet complexe est composé de plusieurs éléments entretenant des rapports entre eux et c'est la gestion de ces rapports qui complexifie l'activité. Ceci est d'autant plus vrai que la tâche complexe invite à l'interdisciplinarité et mobilise toutes sortes de savoirs. Si nous ramenons toutes ces notions à la pratique de l'EO, il s'agira pour l'étudiant de se servir des outils dont il dispose et de s'appuyer sur des compétences qu'il s'est construites pour faire une présentation orale monogérée qui deviendra le résultat visible d'un nombre plus ou moins grand de tâches intermédiaires.

21 Les situations complexes sont propices au développement de compétences. Médioni (2014 : 32-33) explique qu'une situation complexe propose des tâches

«[...] qui font appel à une stratégie de résolution, c'est-à-dire que les apprenants vont devoir combiner non seulement plusieurs procédures utilisant des ressources diverses, mais également user de parcours et de stratégies différentes et avoir une part d'initiative importante, ce qui nécessite des moments de métacognition. [...] La tâche complexe est ouverte et donne lieu à plusieurs réponses possibles, nouvelles, et son résultat n'est donc jamais entièrement prévisible ». 
Pour empêcher que les tâches intermédiaires ne soient atomisées, il faudrait permettre à l'étudiant (Chini, 2008: 64) «de comprendre qu'il a affaire non pas à des microsavoir-faire juxtaposés mais bien à un système dynamique, complexe et hiérarchisé ». La tâche finale que nous étudions est un acte authentique non simulé ${ }^{3}$ réalisable dans des phases successives à travers lesquelles l'étudiant doit passer pour atteindre un apprentissage complet.

En suivant le cadre théorique que nous venons d'esquisser et à partir de nos propres expériences de terrain, il nous semble que, pour rendre plus explicite le modèle didactique de l'EO en LE, il faudrait prendre en considération sept étapes principales dans sa mise en œuvre que nous décrivons sous forme synthétique dans la figure 1.

Figure 1 - Les sept étapes de l'exposé oral

Source : élaboration personnelle

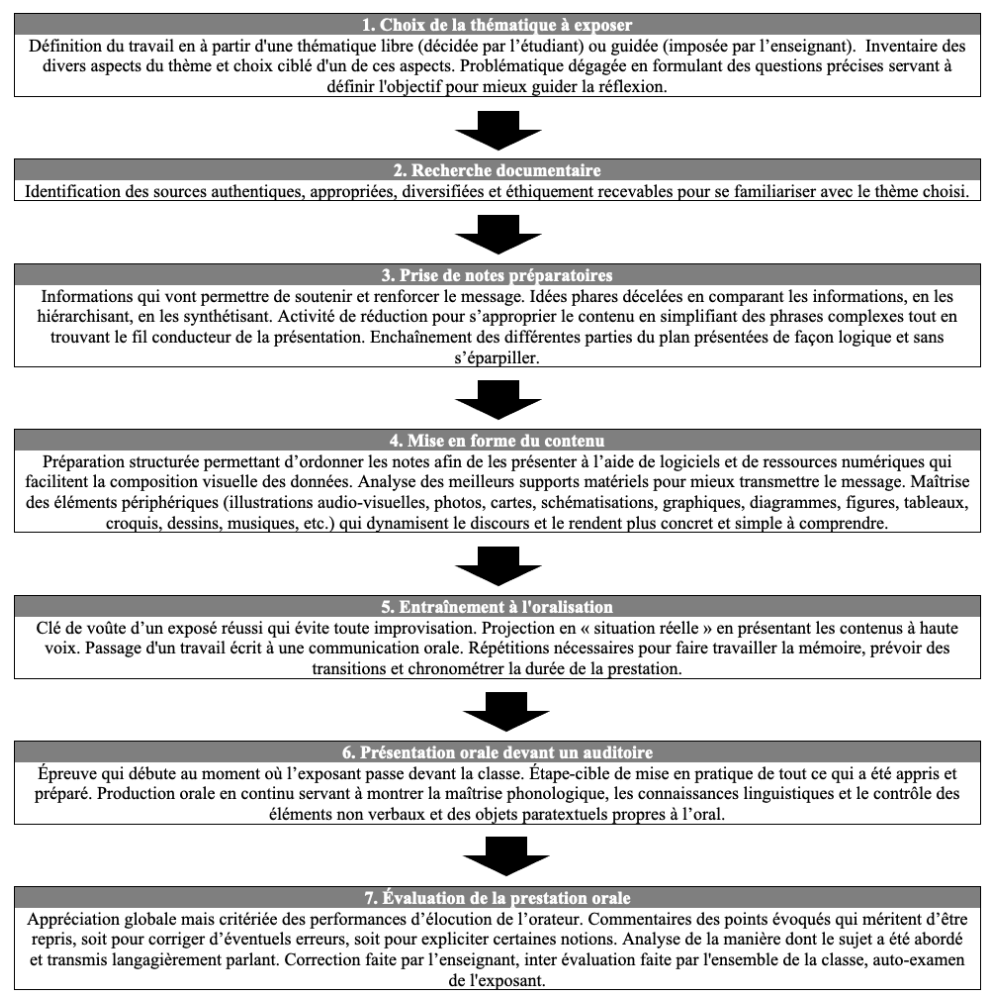

\section{L'exposé oral en langue étrangère aux yeux des étudiants universitaires}

\section{1. Élaboration d'une enquête ad hoc}

Nous avons mené une recherche reposant sur une analyse statistique des données collectées qui émanaient des étudiants ${ }^{4}$. Nous nous sommes basés sur le principe que la mesure quantifiée permet de mettre en évidence les décalages entre, d'une part, les discours et les perceptions qu'ont les étudiants universitaires et, d'autre part, leurs pratiques langagières proprement dites.

L'étude critique relative au cadre conceptuel de l'EO faite dans la partie 2 nous a permis d'élaborer un questionnaire que nous avons utilisé pour interroger une population 
cible possédant certaines caractéristiques qui la rendent relativement homogène. En effet, elle est composée d'individus : i) ayant entre 18 et 25 ans ; ii) appartenant à un conglomérat de formation scientifique et technologique très diversifié et représentatif de l'enseignement supérieur français; iii) étudiant une ou plusieurs LE dans leur formation; iv) se préparant au métier d'ingénieur(e) où l'EO en LE occupe une place incontournable dans l'exercice de la profession ${ }^{5}$.

Le questionnaire proposé aux étudiants était anonyme et directif, c'est-à-dire, comportant des questions fermées. Pour son élaboration, nous avons suivi les préconisations de Blanchet et Chardenet (2011 : 74) pour qui, dans les questions fermées, les réponses sont prédéterminées et que, pour une question, l'informateur n'a qu'un choix limité de réponses prédéfinies sous forme de liste. Nous avons donc exclu les questions ouvertes afin d'éviter le risque de dispersion et d'inexploitabilité des données. Par conséquent, la question fermée est présentée comme un choix unique ou multiple pour lequel l'individu interrogé s'est vu proposer des options de réponses préétablies évitant ainsi des propositions qui puissent s'écarter des informations réellement recherchées. Cette façon de procéder a été utile parce que nous souhaitions interroger un grand nombre d'individus. Le choix du questionnaire directif devenait plus sécurisant car nous étions sûrs que les participants n'allaient pas proposer des réponses biaisées ou relevant d'un hors-sujet puisque les options possibles leur seraient suggérées suivant des critères de pertinence et de faisabilité.

Pour le dépouillage, la méthode adoptée était aléatoire en ce sens que, dans notre population cible, chaque membre avait une chance égale d'être inclus à l'intérieur de l'échantillon. Puisque nous avions une haute probabilité de ne pas mobiliser l'ensemble des élèves-ingénieurs concernés, nous avons donc laissé le hasard décider de la composition de l'échantillon. L'échantillonnage probabiliste nous a permis de mesurer des seuils de confiance aux résultats obtenus, en suivant le principe selon lequel plus l'échantillon est grand, plus l'estimation est précise. Comme ces mesures sont plutôt fiables, nous sommes en condition de faire des inférences au sujet de notre population cible puisque nous considérons que la faible marge d'erreur de notre sondage nous permet de refléter l'opinion de la population globale. Par conséquent, en ce qui concerne la valeur probatoire des résultats obtenus, ceux-ci peuvent parfaitement être considérés comme généralisables à l'ensemble de la population de référence en raison du nombre représentatif des répondants.

Nous proposons dans la figure 2 une visualisation succincte des principaux éléments constitutifs de notre enquête. 
Figure 2 - Présentation de l'enquête «L'exposé oral en LE aux yeux des élèves-ingénieurs » Source : élaboration personnelle

\begin{tabular}{|c|c|}
\hline Public & $\begin{array}{l}\text { Étudiant(e)s de l'Université de Lorraine régulièrement inscrit(e)s en formation initiale ou par } \\
\text { alternance dans l'une des } 11 \text { écoles du Collégium L-INP (proposant une formation sur } 3 \text { ou } 5 \\
\text { ans) ainsi que dans le cycle préparatoire des INP (proposant une formation sur } 2 \text { ans). }\end{array}$ \\
\hline $\begin{array}{l}\text { Composantes } \\
\text { universitaires } \\
\text { concernées }\end{array}$ & $\begin{array}{l}\text { EEIGM : École européenne d'ingénieurs en génie des matériaux } \\
\text { ENIM : École nationale d'ingénieurs de Metz } \\
\text { ENSAIA : École nationale supérieure d'agronomie et des industries alimentaires } \\
\text { ENSEM : École nationale supérieure d'électricité et de mécanique } \\
\text { ENSG : École nationale supérieure de géologie } \\
\text { ENSGSI : École nationale supérieure en génie des systèmes et de l'innovation } \\
\text { ENSIC : École nationale supérieure des industries chimiques } \\
\text { ENSTIB : École nationale supérieure des technologies et industries du bois } \\
\text { POLYTECH : École supérieure des sciences et technologies de l'ingénieur de Nancy } \\
\text { MINES : École nationale supérieure des mines de Nancy } \\
\text { TELECOM : École d'ingénieurs généralistes en informatique et sciences du numérique } \\
\text { PREPA L-INP : cycle préparatoire intégré aux Institut Nationaux Polytechniques }\end{array}$ \\
\hline Objectif & $\begin{array}{l}\text { Recueillir le point de vue des étudiants pour savoir : i) comment les étudiant(e)s pratiquent } \\
\text { l'exposé oral en langue étrangère ; ii) quels sont les bénéfices qu'ils(elles) en tirent ; et iii) } \\
\text { quelles sont les difficultés rencontrées dans la mise en œuvre de cette activité langagière. }\end{array}$ \\
\hline Genre d'enquête & Quantitative et qualitative \\
\hline Type de questionnaire & Anonyme et directif \\
\hline Total de questions & 39 (distribuées en 5 parties) \\
\hline Support & En ligne, sur site dédié, avec recueil automatisé des données \\
\hline Période & 1 mois (du $25 / 04 / 20$ au $25 / 05 / 20)$ \\
\hline Méthode de distribution & Courriel adressé à l'ensemble des étudiants concernés \\
\hline Population cible & 6202 élèves-ingénieurs inscrits pour l'année académique 2019-2020 \\
\hline Nombre de répondants & 492 \\
\hline Échantillon analysé & La totalité des répondants \\
\hline Échantillonnage & Probabiliste \\
\hline Indicateurs & $\begin{array}{l}\text { Pour faciliter leur exploitation, les pourcentages sont arrondis à la moyenne supérieure ou } \\
\text { inférieure. }\end{array}$ \\
\hline Taux de réponse & $8 \%$ \\
\hline Marge d'erreur estimée & $6 \%$ (arrondi correspondant à un niveau de confiance de $99 \%$ ). \\
\hline Valeur probatoire & $\begin{array}{l}\text { Résultats généralisables à l'ensemble de la population de référence en raison du nombre } \\
\text { représentatif des répondants }\end{array}$ \\
\hline
\end{tabular}

\subsection{Analyse et discussion des résultats}

29 Notre enquête sur l'EO en classe de LE montre les répercussions positives d'une telle expérience sur le sentiment d'efficacité personnelle dans la production orale en continu des étudiants. Dans les paragraphes suivants, une fois analysée globalement la manière dont ils « pratiquent » l'EO, nous allons voir quels sont les bénéfices qu'ils en tirent.

\subsubsection{Les pratiques des étudiants pour l'exposé oral en langue étrangère}

30 La totalité des participants (100 \%) déclare avoir déjà fait un EO en LE. Ceci prouve qu'il s'agit d'une activité très fréquente en milieu universitaire. Au cours de leur formation, presque la moitié des élèves-ingénieurs ( $48 \%)$ affirme avoir déjà fait entre une et six prestations de ce genre. En règle générale, ils sont majoritaires $(69 \%)$ à aimer cette activité d'expression orale en continu qu'ils voient comme étant vraiment profitable pour améliorer leurs performances en LE (82 \%). Ceci se vérifie encore au niveau de leur ressenti par rapport à la progression de leurs apprentissages puisqu'une grande partie d'entre eux (71\%) déclare faire des progrès en LE depuis qu'ils font des EO.

31 Les étudiants enquêtés sont censés les faire dans plusieurs LE. Cependant, les quatre langues dans lesquelles ils travaillent le plus l'EO sont, dans l'ordre, l'anglais (99\%), l'espagnol (42\%), l'allemand (35\%) et le français (14\%). Ces chiffres doivent être nuancés à la baisse lorsqu'il s'agit de vérifier dans quelles LE ils se sentent le mieux préparés pour faire des exposés oraux. D'après leurs réponses, s'ils sont plus opérationnels en anglais (90\%), ils le sont beaucoup moins en espagnol (15\%), en 
français (13\%) et en allemand (9\%), ce qui rendrait nécessaire un travail plus fréquent de la production orale en continu dans d'autres LE différentes de l'anglais.

Selon les avis des participants, l'EO en LE peut être introduit comme activité de classe dans tous les niveaux de langue mais ils indiquent clairement qu'ils préfèreraient réaliser ce type d'activités dans les programmes de niveau intermédiaire B1-B2 (57\%), de niveau avancé C1-C2 (33\%) et, dans une moindre mesure, de niveau initial A1-A2 (29 $\%)$.

Les trois quarts des étudiants $(75 \%)$ expriment être satisfaits par rapport à leur performance en matière de présentation orale. Néanmoins, leur aptitude à s'adresser à un auditoire varie selon la langue. En ce qui concerne leur première LE, ils déclarent, pour une bonne partie (62\%), pouvoir développer un EO clair sur un sujet préparé dans leur domaine, en avançant des raisons pour ou contre un point de vue particulier, en présentant les avantages et les inconvénients d'options diverses, en prenant en charge une série de questions après l'exposé avec un degré d'aisance et de spontanéité qui ne cause pas de tension. Ces habiletés correspondent à un niveau intermédiaire B2-B1. Pour leur deuxième LE, plus de la moitié (58 \%) annonce pouvoir faire un exposé simple, préparé à l'avance, sur un sujet familier dans leur domaine, assez clair pour être suivi sans difficulté et dans lequel les points importants sont expliqués avec précision. Ils peuvent également gérer les questions qui suivent à condition de se faire aider éventuellement pour formuler une réponse. Ces capacités correspondent à un niveau intermédiaire plutôt B1, voire à un niveau élémentaire A2.

L'activité est perçue comme étant chronophage par plus de la moitié (52\%) des enquêtés. Habituellement, pour préparer un EO en LE, la plupart d'entre eux $(70 \%)$ ont besoin de trois à cinq heures de travail. Par ordre d'importance, ils semblent préférer traiter certains types de sujets: les thèmes en lien avec l'actualité (57\%) ou les disciplines qu'ils étudient ( $55 \%$ ) aussi bien que les thématiques de la vie quotidienne (44\%) en même temps que celles en relation avec leur futur métier (41\%). Un détail attire notre attention quant à la préparation de l'EO : les personnes sondées déclarent majoritairement (66 \%) utiliser leur LE ainsi que leur langue maternelle, ce qui peut complexifier la tâche car elles semblent se priver des avantages du travail exclusif en langue cible.

\subsubsection{Les bénéfices de l'exposé oral en langue étrangère en matière de compétences}

Sur le plan des compétences linguistiques, l'EO en LE est une activité assez complète. Cependant, bien qu'elle permette d'entretenir son niveau de langue et d'accroître le degré de maitrise lexicale, grammaticale et syntaxique, l'essentiel de ses bienfaits se trouve, d'après les étudiants qui ont participé à cette enquête, au niveau phonologique. Plus des trois quarts des enquêtés $(78 \%)$ déclarent que c'est une tâche idéale pour travailler la prononciation. D'autres affirment que l'exposé est utile pour travailler l'intonation (58\%), pour apprendre à ajuster le rythme (55\%) ou pour résoudre un problème d'accent ( $40 \%)$.

Par rapport aux compétences communicatives, plus de la moitié des enquêtés trouve que l'EO en LE leur permet de s'entraîner à mieux communiquer à l'oral de façon continue (54\%) et à captiver un auditoire en retenant l'attention de ce dernier (54\%). La moitié d'entre eux (50 \%) profite de cette tâche pour s'exercer à reformuler dans une 
intention explicative. Cela leur permet de progresser dans l'acquisition d'expressions utiles (48\%) en développant leurs capacités langagières et discursives (45\%). Certains s'appuient sur l'exposé pour libérer leur parole (44\%) ou pour réfléchir à leur langage corporel quand ils parlent (44\%).

L'EO en LE est surtout une tâche plus qu'appropriée pour développer des compétences transversales. Parmi celles qui ont été les plus citées par les étudiants, nous trouvons : apprendre à élaborer un diaporama attrayant $(59 \%)$; augmenter son niveau de confiance $(48 \%)$ notamment en s'attaquant à sa timidité $(48 \%)$; apprendre à gérer le trac paralysant $(42 \%)$ en dépassant ses peurs $(38 \%)$; autoévaluer son niveau de performance langagière $(41 \%)$ en restructurant les informations dans un plan cohérent (40\%). Plus d'un tiers des sondés déclare développer des capacités pour reformuler un discours écrit en un discours oral clair et accessible (38\%) en examinant sa manière de parler (33\%) et en corrigeant les faiblesses repérées (34\%).

Si nous savions que les compétences à développer grâce à l'EO étaient vraiment nombreuses, nous voulions connaître de façon plus précise quelles étaient les capacités développées lors de chacune des sept étapes (cf. figure 1) qui rythment cette activité. Ainsi, les étudiants ont confirmé que, pendant la préparation d'un EO en LE, les compétences développées dans la phase initiale concernant le choix de la thématique à traiter sont concrètement celles qui se basent sur les savoirs servant à définir l'objectif d'un exposé (64\%). Ces capacités leur permettent de limiter un sujet complexe (60\%) pour en dégager une problématique (61\%) ainsi qu'activer des connaissances initiales (38 \%) pour inventorier les divers aspects d'un thème (37 \%).

Dans l'étape concernant la recherche documentaire, plus de la moitié des personnes sondées ont indiqué qu'elles développent les savoir-faire suivants: confronter les informations afin de les relativiser et de les articuler (61\%), savoir chercher des sources éthiquement recevables (52\%) en repérant l'information utile et les noyaux de sens (50\%), autrement dit, savoir reconnaître l'information pertinente dans la masse documentaire ( $48 \%$ ) en évaluant la fiabilité et l'authenticité des références trouvées (51 $\%)$. Un certain nombre de sondés acquièrent également des savoirs leur permettant de questionner les sources avec un esprit critique (45\%) en distinguant entre l'interprétation d'un fait ou point de vue et un fait ou donnée objective (41\%).

En ce qui concerne la prise des notes préparatoires, cette tâche est bénéfique en ce sens qu'elle permet de développer des savoirs en vue de hiérarchiser l'information pour faire ressortir les idées phares (70 \%), trouver le fil conducteur de sa présentation (59 $\%)$ en structurant un discours de façon claire (54\%) et en sachant réduire des phrases complexes ( $49 \%)$. Un pourcentage significatif des réponses a permis de corroborer que cette étape est utile pour comparer des informations ( $42 \%)$ dans le but d'élaborer un plan chronologique (37 \%) en relevant avec précision des éléments chiffrés ou factuels (33\%).

41 Pendant la mise en forme du contenu de l'EO, les savoir-faire à déployer sont encore bien précis. Les étudiants en ont choisi les suivants : savoir mettre en évidence des idées (64\%) tout en présentant un travail cohérent et ordonné (58\%); savoir choisir des mots-clés en lien avec le sujet abordé ( $57 \%$ ) en réduisant les longs contenus à de courtes expressions (54\%); savoir veiller à l'homogénéité visuelle d'un exposé (54\%) en utilisant un logiciel pour réaliser des présentations multimédias (49\%) permettant l'intégration des ressources iconographiques (47\%). 

la perspective pragmatico-discursive, ce qui implique la prise en compte des traits distinctifs d'un type de discours socialement pertinent et professionnellement utile. L'oral, en tant qu'objet d'enseignement, gagnerait donc à se concentrer sur des genres de discours particuliers, dans notre cas l'EO, présents aussi bien à l'intérieur qu'à l'extérieur de la salle de classe. Ainsi, grâce aux différentes données scientifiques utilisées dans cet article, provenant des références théoriques et du cadre applicatif, nous avons mis en exergue que l'EO en LE est une tâche propice pour étayer l'apprentissage de l'oral.

Grâce aux résultats fournis par l'enquête ad hoc, nous avons pu vérifier les hypothèses émises dans notre introduction. Non seulement l'EO en LE est une activité stimulante pour diminuer le niveau de lathophobie mais elle s'érige en une tâche formelle d'enseignement-apprentissage permettant de développer des facultés très diverses. Nous avons pu vérifier que, en milieu universitaire, il s'agit d'une activité fréquente, appréciée par les étudiants et qui est à l'origine de nombreux progrès linguistiques. Cependant, même si cette activité peut être réalisée à tous les niveaux de langue 
référencés par le CECRL, il semblerait qu'elle soit plus adaptée pour les niveaux intermédiaires et supérieurs.

Les étudiants restent convaincus de l'effet de levier important que recouvre l'EO pour l'apprentissage d'une LE car il apporte de nombreux bénéfices en facilitant le développement de certaines habiletés. Parmi les capacités signalées figurent, en premier lieu, les compétences linguistiques dans leur volet essentiellement phonologique (travail sur la prononciation, l'intonation, le rythme, l'accent). Sans oublier les aspects lexicaux, grammaticaux et syntaxiques qu'on travaille forcément en faisant un EO, l'essentiel des bienfaits de cette tâche se trouve au niveau phonétique. Viennent ensuite les compétences communicatives développées grâce à une prestation orale qui permet d'améliorer ses capacités discursives pour reformuler dans une intention explicative, pour captiver un auditoire en s'appuyant sur le langage nonverbal. En troisième place, suivent les compétences transversales liées notamment à une augmentation du niveau de confiance du locuteur, à une diminution de la timidité et du trac paralysant.

Notre étude montre bien que les sept étapes que nous proposons comme protocole de travail sont propices au développement des compétences. Lors de l'étape initiale où il est question de choisir la thématique à aborder, on apprend à définir un objectif en limitant un sujet complexe pour en dégager une problématique. Dans l'étape de recherche documentaire, on développe la capacité à s'appuyer sur des sources fiables pour confronter et articuler des informations en reconnaissant les données pertinentes dans la masse documentaire. L'étape de prise de notes préparatoires sert à comparer et à hiérarchiser l'information à partir d'un fil conducteur. L'étape suivante de mise en forme apporte aussi son lot de bénéfices car elle s'avère utile pour construire une présentation multimédia ainsi que pour structurer son discours le plus clairement possible dans un plan cohérent où les idées clés seront mises en évidence grâce à l'intégration de ressources iconographiques. L'entrainement à l'oralisation est une phase inéluctable de la préparation pendant laquelle on apprend à gérer son expression orale en contrôlant son temps et son rythme, ainsi qu'à s'exprimer à partir de notes minimales tout en travaillant sa mémoire. L'étape de confrontation à un auditoire est, sans conteste, celle où les apprentissages sont les plus évidents car s'exprimer efficacement devant un public implique adopter une posture stable, parler fort et de façon intelligible en modulant sa voix, retenir l'attention, réagir sans paniquer, occuper l'espace en gérant sa gestuelle. L'étape finale concernant l'évaluation sert aussi à développer des compétences non moins utiles comme, par exemple, se remettre en question en acceptant une remarque justifiée, identifier et corriger des erreurs et, enfin, estimer l'efficacité d'une prestation orale.

Pour légitimer la pratique réfléchie de l'EO chez les étudiants, nous pensons qu'il est temps d'organiser cet enseignement de façon explicite en adoptant une méthodologie propre à ce genre discursif. Cela implique d'aménager des moments, nombreux et réguliers, pendant lesquels les étudiants auront l'occasion de s'exprimer, de s'écouter et d'être évalués. L'EO devient ainsi une activité qui s'apparente à un emboîtement de tâches complexes de telle sorte que la réussite de la première étape conditionne largement la réussite des étapes suivantes.

50 Finalement, nous l'avons bien compris, mener une prestation orale sans retenue ni complexes est un exercice qui ne s'improvise pas, d'où l'intérêt d'inclure ce genre d'activités dans un programme résolument centré sur les besoins des étudiants. Cette 
tâche complexe qu'est l'EO en LE se présente donc comme une démarche adaptée pour asseoir des compétences en mobilisant, à des niveaux différents de maîtrise, des connaissances et des attitudes. Les enjeux de l'EO en LE sont définitivement ceux des avantages que cette activité procure. La prise de parole devant un public développe en fait des capacités qui vont dans le sens d'une plus grande autonomie des étudiants. Nous pouvons conclure que l'EO en LE est une activité langagière complexe. Compte tenu de cette complexité, nous proposons qu'elle soit sérieusement envisagée dans les curriculums d'enseignement-apprentissage des LE au niveau universitaire en adoptant la méthodologie de travail explicite mise en lumière dans cette contribution pour, in fine, mieux développer des compétences d'une haute valeur ajoutée.

\section{BIBLIOGRAPHIE}

Alrabadi, E. (2011). Quelle méthodologie faut-il adopter pour l'enseignement/apprentissage de l'oral ? Didáctica, Lengua y Literatura, $\mathrm{n}^{\circ} 23$, p. 15-34.

Bourguignon, C. (2006). De l'approche communicative à l'approche communic-actionnelle : une rupture épistémologique en didactique des langues-cultures. Synergies Europe, n 1, p. 58-73.

Blanchet, P. et Chardenet, P. (2011). Guide pour la recherche en didactique des langues et des cultures. Approches contextualisées. Éditions des archives contemporaines.

Bronckart, J.-P. et Dolz J. (1999). La notion de compétence : quelle pertinence pour l'étude de l'apprentissage des actions langagières ? L'énigme de la compétence en éducation. De Boeck, p. 27-44.

Conseil de l'Europe. (2001). Cadre européen commun de référence pour les langues : apprendre, enseigner, évaluer. Unité des politiques linguistiques du COE.

Conseil de l'Europe. (2018). Cadre européen commun de référence pour les langues : apprendre, enseigner, évaluer. Volume complémentaire avec de nouveaux descripteurs. Unité des politiques linguistiques du COE.

Crosnier, E. et Décuré, N. (2018). Parler ou ne rien dire : La prise de parole en classe d'anglais Lansad. RDLC-Recherches en didactique des langues et des cultures, $\mathrm{n}^{\circ} 15-2$. Disponible sur : http://journals.openedition.org/rdlc/3062

Chini, D. (2008). Objectif méthodologique : construction d'une mémoire procédurale? Psycholinguistique et didactique des langues étrangères. Ophrys.

Dolz, J. et Scheuwly, B. (1998). Pour un enseignement de l'oral : initiation aux genres formels de l'oral. ESF éditeur.

Gautier, S. (2017). Comment faire de l'exposé oral un vrai moment d'apprentissage ? MÉTHODALRevue internationale de méthodologie de l'enseignement/apprentissage des langues, vol. 1 , p. 229-245.

Kerbrat-Orecchioni, C. (1999). La conversation. Seuil. 
Manolescu, C. (2013). L'expression orale en milieu universitaire. Synergies Roumanie, $\mathrm{n}^{\circ} 8$, p. 109-121.

Médioni, M.-A. (2014). La langue, vivante. Cahiers pédagogiques des CRAP, n 510, vol. : Des tâches complexes pour apprendre, p. 32-34.

Miecznikowski, J., Mondada, L., Müller, K. et Pieth, C. (2001). L'exposé scientifique comme activité pratique et interactive. Les Carnets du Cediscor (Centre de Recherches sur les Discours Ordinaires et Spécialisés), $\mathrm{n}^{\circ}$ 7. Disponible sur : http://journals.openedition.org/cediscor/313

Musial, M., Pradère, F. et Tricot, A. (2012). Comment concevoir un enseignement? De Boeck Supérieur, 1ère édition.

Nissen, E. (2011). Variations autour de la tâche dans l'enseignement/apprentissage des langues aujourd'hui. ALSIC-Apprentissage des Langues et Systèmes d'Information et de Communication, vol. 14. Disponible sur : http://alsic.revues.org/2344

Richer, J.-J. (2014). Conditions d'une mise en œuvre de la perspective actionnelle en didactique des langues. Recherche et pratiques pédagogiques en langues de spécialité, vol. XXXIII, $\mathrm{n}^{\circ} 1$, 33-49.

Tano, M. (2021, sous presse). Analyse typologique des erreurs de production orale en continu en langue étrangère, EDL-Études en Didactique des Langues : revue du Laboratoire Interuniversitaire de Recherche en Didactique Lansad, no 36.

Tian, N. (2016). La formation méthodologique de l'exposé oral au niveau débutant. Synergies Chine, $\mathrm{n}^{\circ} 11$, p. 89-100.

Vaissière, J. (1999). Utilisation de la prosodie dans les systèmes automatiques : un problème d'intégration des différentes composantes. Faits de langue, p. 9-16. Éditions Ophrys.

Zahnd, G. (1998). L'exposé oral, un outil pour transmettre des savoirs. Repères, vol. L'oral pour apprendre, $\mathrm{n}^{\circ} 17$, p. 41-54.

\section{NOTES}

1. Peur de se tromper, de commettre des fautes de traduction, d'orthographe, de grammaire. En règle générale, plus le niveau de langue est bas, plus les étudiants sont mal à l'aise avec l'idée de tenir un discours en public.

2. «Complexe » ne veut pas dire « compliqué ».

3. Au sens donné par Tian (2016:91) lorsqu'elle affirme que l’EO « [...] n'est plus une simulation, mais une action réelle avec un objectif de communication bien précis ».

4. Cette recherche a eu lieu à l'Université de Lorraine dans 11 composantes appartenant au Collégium Lorraine-Institut National Polytechnique et formant de futurs ingénieurs.

5. Dans une enquête précédente, faite par nos soins auprès d'un échantillon d'ingénieurs actifs (319 répondants), nous avions conclu que la réussite professionnelle mobilise souvent des talents en lien avec l'aptitude à la communication. Dans ce contexte, $69 \%$ des ingénieurs déclarent avoir réalisé des exposés en LE. 


\section{RÉSUMÉS}

L'exposé oral en langue étrangère est une activité fréquente dans l'enseignement supérieur mais sa pratique gagnerait à être améliorée si nous voulons qu'il ne soit pas vu comme un pur artefact universitaire. Pour y parvenir sans improvisation, il faudrait savoir quelles sont les caractéristiques qui définissent la production orale en continu. Car l'exposé oral, en tant que genre discursif hautement formalisé, se réalise en suivant des étapes précises et séquencées qui permettent de découper une tâche complexe en sous-tâches formatrices et autonomisantes. Une pédagogie propre à l'exposé oral semble nécessaire pour tirer pleinement parti de cette activité exigeante, souvent stimulante mais toujours redoutée par les étudiants. La présente recherche a donc pour but de mettre en lumière les pratiques rapportées autour de cette activité grâce à une enquête menée auprès d'un échantillon représentatif d'étudiants universitaires. Cette étude exploratoire a permis d'analyser, étape par étape, les bénéfices que, selon les étudiants, procure l'exposé oral. Les données obtenues ont montré qu'il s'agit d'un moyen idéal permettant d'améliorer diverses habiletés linguistiques et communicatives et de développer des compétences transversales d'une grande utilité. L'étude conclut que, pour faire de l'exposé oral un objet enseignable et apprenable, une pratique méthodologique s'inspirant des théories de l'enseignement explicite permettrait aux étudiants d'acquérir une réelle autonomie dans le développement de leurs compétences de production orale en langue étrangère.

Oral presentation in a foreign language is a frequent activity in higher education, but its practice would need to be improved if we are not to see it as a pure academic artefact. To achieve this without improvisation, it would be necessary to know what are the characteristics that define continuous oral production. Because the oral presentation, as a highly formalized discursive genre, is carried out by following precise and sequenced steps which allow a complex task to be broken down into formative and empowering sub-tasks. A pedagogy specific to oral presentation seems necessary to take full advantage of this demanding activity, often stimulating but always feared by students. The purpose of this research is therefore to shed light on the practices reported around this activity thanks to a survey conducted among a representative sample of university students. This exploratory study allowed for a step-by-step analysis of the benefits that the students believe the oral presentation provides. The data obtained has shown that this is an ideal way to improve various linguistic and communication skills and to develop useful transversal skills. The study concludes that, in order to make the oral presentation a teachable and learnable object, a methodological practice inspired by the theories of explicit teaching would allow students to acquire real autonomy in the development of their speaking skills in a foreign language.

\section{INDEX}

Keywords : oral communication, competence, oral presentation, oral methodology, complex task Thèmes : Varia

Mots-clés : communication orale, compétence, exposé oral, méthodologie de l'oral, tâche complexe 


\section{AUTEUR}

\section{MARCELO TANO}

Enseignant titulaire à l'Université de Lorraine

Docteur qualifié en sciences du langage et études romanes

Chercheur associé du Laboratoire Inter-universitaire de Recherche en Didactique Lansad L'auteur pratique l'exposé oral en milieu universitaire depuis plus de deux décennies et s'intéresse tout particulièrement aux méthodologies actives d'enseignement des langues étrangères à visée académique et professionnelle.

mtano[at]lairdil.fr 